Michael D. Cusimano, M.D., M.H.P.E., F.R.C.S.(C),

Paul Ohori, M.D., A. Julio Martinez, M.D., Charles

Jungreis, M.D., and Donald C. Wright, M.D.

\title{
Pituitary Carcinoma
}

\begin{abstract}
The presence of distant metastases may be asymptomatic in patients who present with symptoms and signs due to the local mass effects of an invasive pituitary adenoma. A case of pituitary carcinoma in a 54-year-old man who presented with widespread asymptomatic distant metastases 12 years after initial diagnosis is reviewed. The long course and asymptomatic metastases suggested a relatively slow-growing malignancy. The factors that played a role in the pathogenesis of the metastasis are unknown. A review of the literature on pituitary carcinoma suggests that accurate diagnosis and a multidisciplinary approach to management of such lesions emphasizing surgery, radiotherapy, and hormonal manipulation may provide these patients with the longest and best quality of survival. (Skull Base Surgery, 4(1):46-51, 1994)
\end{abstract}

Although pituitary adenomas represent 6 to $15 \%$ of all intracranial neoplasms, true pituitary carcinoma has been rarely reported in the literature. In contrast to pituitary adenomas, pituitary carcinomas are more commonly nonfunctioning. ${ }^{\prime}$ Of the 46 reported cases, six patients had acromegaly, eight had Cushing's disease, nine had hyperprolactinemia, and the remaining patients were reported to have nonsecreting tumors. ${ }^{1-3}$ Furthermore, due to the lack of specific markers, the diagnosis of pituitary carcinoma remains difficult prior to demonstration of metastasis. In view of the rarity of this disease, treatment is often difficult to plan. This case report highlights issues of the diagnosis and management of pituitary carcinoma and presents the first information on the proliferative capacity of pituitary carcinoma.

\section{CASE REPORT}

A 54-year-old man presented in 1979 with symptoms compatible with hypopituitarism, occasional nose bleeds, and a change in his left visual field. Investigation revealed a large sellar mass, and in March 1980 a hemorrhagic and cystic mass was excised via a transcranial approach. Postoperatively, he received 5400 cGy conventional teletherapy. The patient did well, with only mild symptoms, until 1989, when he developed lethargy and dizziness and repeat investigation disclosed a recurrence. He had a debulking procedure via transsphenoidal route in 1989 , followed by treatment with somatostatin. The patient's magnetic resonance imaging (MRI) scans remained stable until late 1990.

In December 1990, he developed symptoms of nasal obstruction, decreased vision in the left eye, and hearing loss on the right. An MRI scan demonstrated progression of disease with carotid encasement and cavernous sinus invasion bilaterally. He lost 20 pounds of weight over the next few months. On presentation to our center, examination revealed visual acuity of 20/50 on the left and 20/25 on the right, limited up gaze of the right eye, and diplopia on up gaze. In addition to hypesthesia in the right $\mathrm{V}_{2}$ region and House grade III-type weakness of the facial muscles on the right side, the patient was also deaf in the right ear. The rest of the neurologic examination was intact, and his general physical examination was normal.

Repeat MRI scanning revealed a large sellar and suprasellar tumor extending through the clivus, sphenoid and ethmoid sinuses superiorly, into the suprasellar cistern to displace the optic chiasm (Fig. 1). The tumor involved both cavernous sinuses and encased both cavernous carotid arteries, but it did not appear to occlude them. Posteriorly, the tumor extended to the right petrous apex and through the clivus into the prepontine cistern, displacing the brainstem and basilar artery. It extended also into the right cerebellopontine angle and into the right internal auditory canal. Inferiorly, the tumor extended into the right jugular fossa and right hypoglossal canals and extracranially, it infiltrated behind the right longus coli muscle.

Skull Base Surgery, Volume 4, Number 1, January 1994 Departments of Neurological Surgery (M.D.C., D.C.W.), Pathology (P.O.), Neuropathology (A.J.M.), and Radiology (C.J.), University of Pittsburgh School of Medicine, Presbyterian University Hospital, Pittsburgh, Pennsylvania Reprint requests: Dr. Cusimano, Division of Neurosurgery, St. Michael's Hospital, University of Toronto, 38 Shuter Street, Toronto, Ontario, Canada 


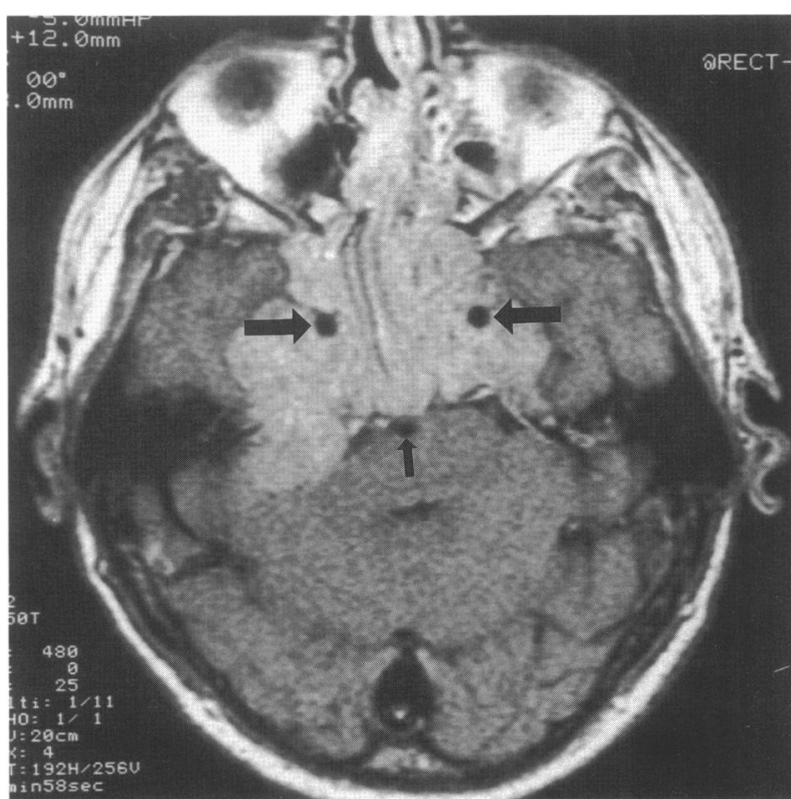

Figure 1. Contrast enhanced axial magnetic resonance scan ( $T R=400, T E=25)$. The tumor encases but does not occlude both internal carotid arteries (arrows) as it invades both cavernous sinuses. The basilar artery (short arrow) is compressed against the pons, which is also deformed by the mass. The clivus has been destroyed by the mass and there is extension into the sphenoid and ethmoid sinuses.

Angiographically, the tumor was extremely vascular (Fig. 2) and demonstrated early venous drainage. The right sigmoid sinus was occluded. No vessels could be found that could be safely embolized and the patient clinically failed a balloon test occlusion of the right internal carotid artery.

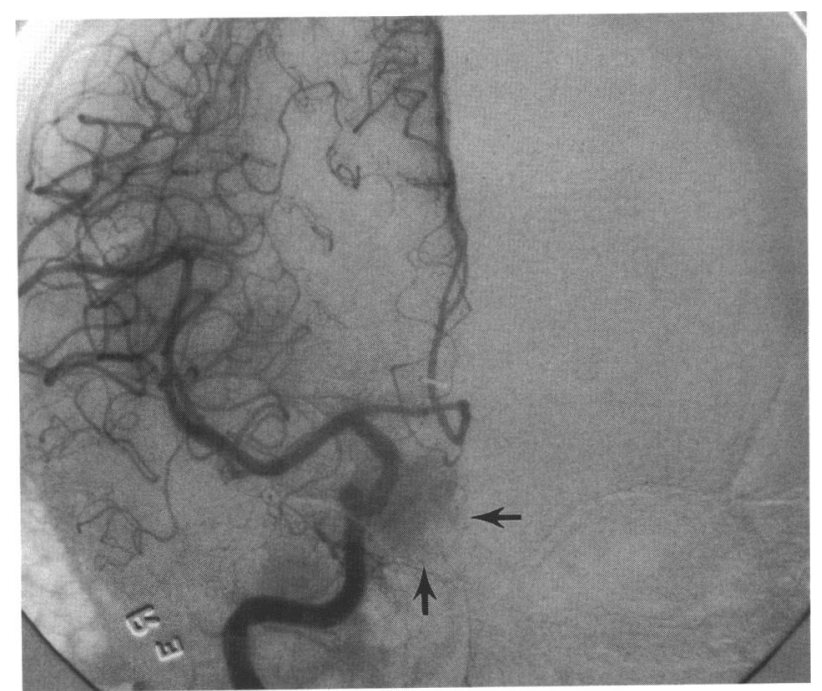

Figure 2. Anteroposterior view of a right internal carotid artery angiogram. The internal carotid artery is patent but a significant tumor blush (arrows) is apparent, which is not typical of adenoma.
Radiologic imaging of the chest incidentally revealed a lesion of the left tenth rib (Fig. 3A, B) and a bone scan revealed lesions of the left tenth rib, sacroiliac joint, lumbar spine, and multiple calvarial sites. Palliative debulking of the optic tract, chiasm, and paranasal sinuses was performed to relieve his primary symptoms via transbasal subfrontal approach using a bifrontal and right temporal craniotomy and orbitozygomatic osteotomy. A biopsy of the left tenth rib was also taken. Postoperatively, the patient was restarted on somatostatin, and he received 2000 cGy of conventional radiation to his left tenth rib. His tumor has not changed in size and he has remained clinically stable during the 16 months since his last surgery.

\section{PATHOLOGIC FINDINGS}

Microscopic examination of the original lesion from the pituitary gland revealed a neoplasm with marked cellular atypism, nuclear pleomorphism, and occasional bizarre, enlarged, and hyperchromatic nuclei with prominent nucleoli (Fig. 4). The recurrent adenomas from 1989 and 1990 as well as the metastatic rib lesion (Fig. 5) showed similar pleomorphic and atypical features. The most recently recurrent pituitary lesion disclosed similar histologic features as the original neoplasm. Ultrastructurally, the neoplastic cells were composed of sheets of polygonal cells with a few electron-dense neurosecretory granules; the chromatin was finely dispersed and nuclei typically exhibited prominent nucleoli (Fig. 6). Immunohistochemical staining for the proliferating cell nuclear antigen (PCNA) cyclin was performed and compared to a typical pituitary adenoma. There were no significant differences in the number of proliferating cells.

\section{DISCUSSION}

\section{Diagnosis}

Most pituitary adenomas grow by expansion and displacement of adjacent structures. Approximately $10 \%$ demonstrate gross invasion and cytologic features suggesting rapid proliferation (particularly mitotic activity). In the past, many pituitary carcinomas were diagnosed on the basis of local invasion or marked cellular atypism. However, adenomas can display varying degrees of polymorphism, invasiveness, mitotic activity, and cellular atypia, ${ }^{4}$ and it has been suggested that microscopic criteria cannot be used to differentiate pituitary adenomas from carcinomas. ${ }^{5}$ Those tumors with local destruction of bone and soft tissue and extension through the meninges are classified as invasive adenomas, $, 6,7$ whereas, those adenomas with distant or cerebrospinal metastasis are called pituitary carcinoma. Three cases have been reported of pituitary carcinoma in the absence of cytologic features of malignancy. $5,8,9$ 
A
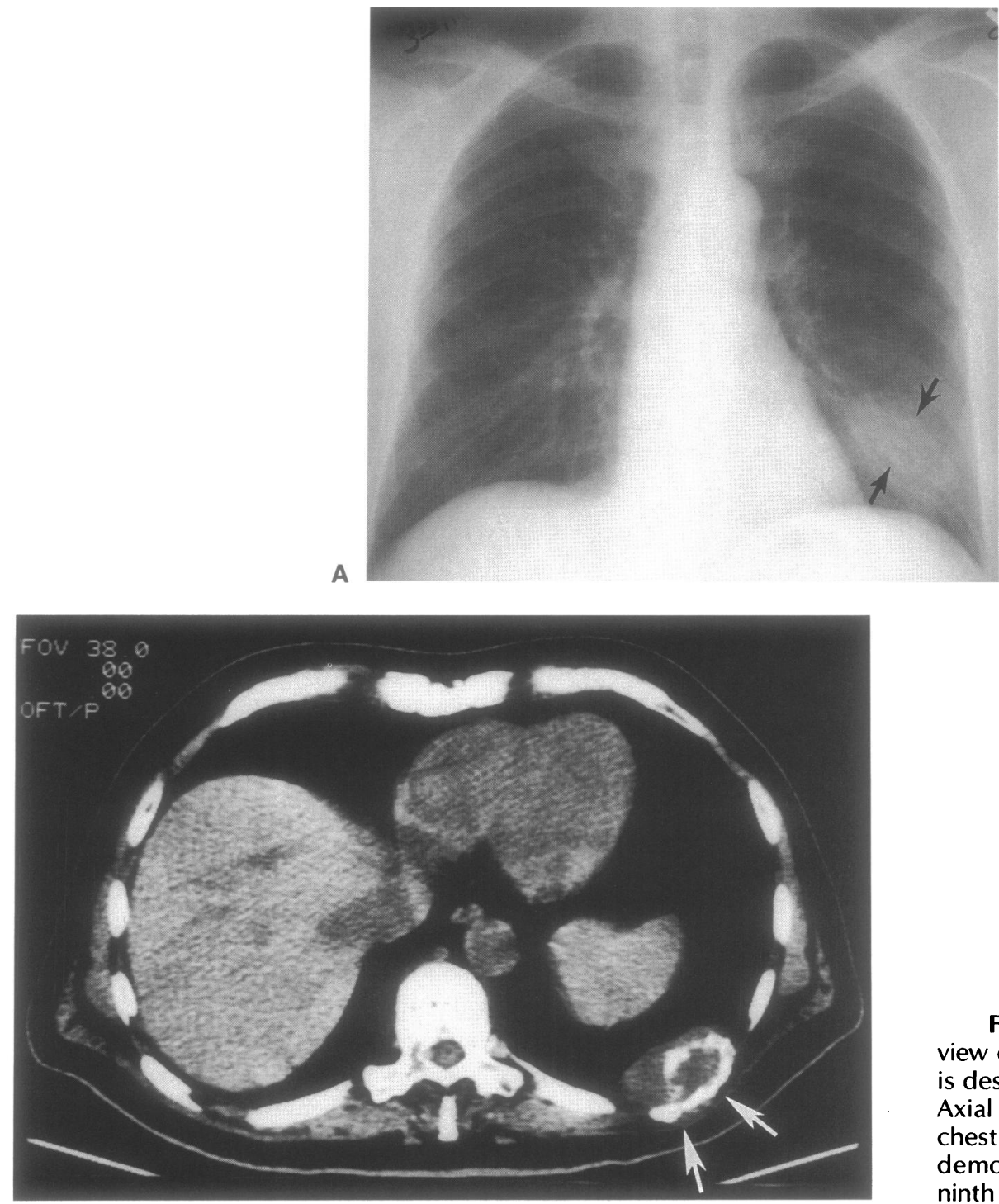

Figure 3. A: Anteroposterior view of the chest. The left ninth rib is destroyed posteriorly (arrows). B: Axial computed tomography of the chest. A destructive mass (arrows) is demonstrated in the posterior left ninth rib.

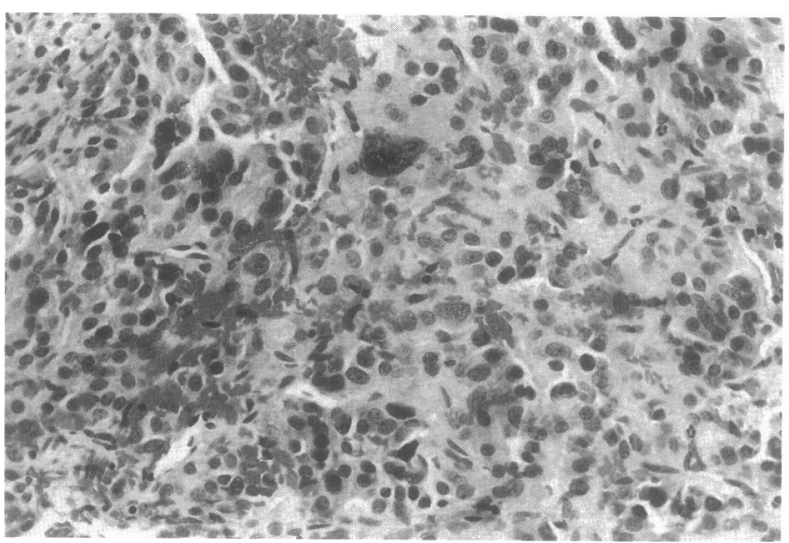

Figure 4. Photomicrograph of the original pituitary lesion, showing marked cellular atypism with nuclear pleomorphism and hyperchromatism with prominent nucleoli. $(\mathrm{H} \& \mathrm{E} ; \times 250$.

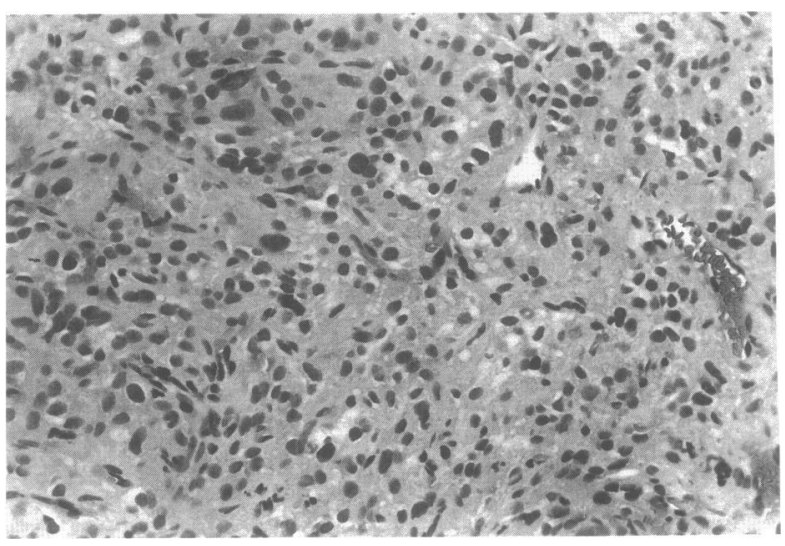

Figure 5. The metastatic lesion from the left rib shows histopathologic features similar to the original and recurrent neoplasms. (H\&E; $\times 250$.) 


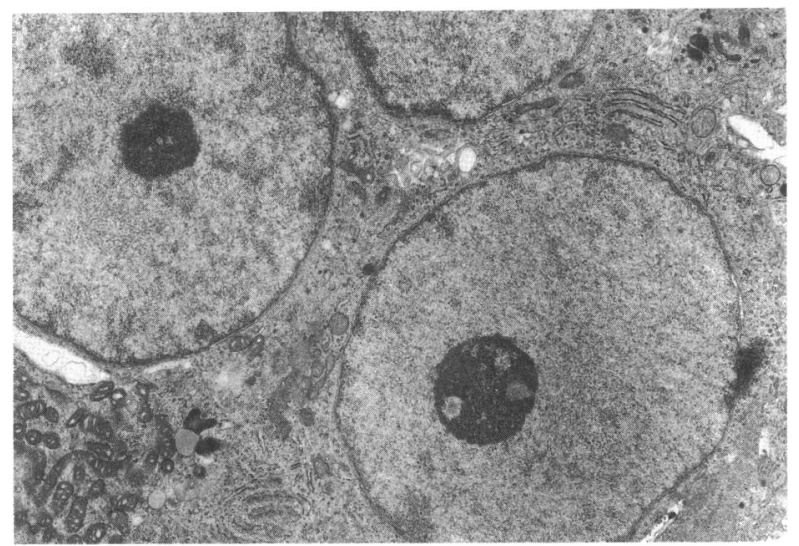

Figure 6. Electron micrographs from the most recent recurrent pituitary neoplasm characterized by large polygonal cells with prominent nuclei and conspicuous nucleoli and a scant number of small secretory granules. (EM: $\times 6300$.)

Faced with a possible pituitary carcinoma, it is essential to exclude metastatic carcinoma because it may mimic a pituitary adenoma, and thus be difficult to differentiate from a sellar tumor. ${ }^{10}$ Particular confusion may be encountered when a carcinoma exhibiting neuroendocrine features, such as an islet cell neoplasm, metastasizes to the sella. In these cases, immunohistochemical and ultrastructural studies may be of assistance in establishing the proper diagnosis. " Neuroimaging is generally nonconclusive in distinguishing pituitary metastasis from adenomas. ${ }^{12,13}$ In our patient, the diagnosis of pituitary carcinoma was established by the presence of metastasis and an invasive intracranial lesion. It is extremely unlikely that a patient with a primary metastatic adenocarcinoma to the pituitary would survive for 13 years.

Although cell kinetic information has proved useful in the evaluation of tumor progression and long-term outcome in breast carcinoma ${ }^{14}$ and several central nervous system tumors, ${ }^{15}$ information on human pituitary adenomas has only recently become available. Hsu et al ${ }^{16}$ studied 30 patients with recurrent pituitary adenomas, 32 nonrecurrent adenomas, and seven normal pituitary tissue samples. Mean percentages of PCNA positive tumor nuclei of the recurrent adenomas (both at initial presentation and at recurrence) were significantly higher than those of nonrecurrent tumors. A higher PCNA index was positively correlated with larger tumors, those with extrasellar extension, those that were incompletely excised, and with shorter disease-free survival in Hsu et al.'s study. Our study is the first to provide information of the proliferative capacity of pituitary carcinoma and did not confirm the findings of Hsu et al.

In three fourths of reported cases of pituitary carcinoma, metastatic disease was not suspected at the time of presentation, and a diagnosis of pituitary carcinoma in general was not established until postmortem demonstration of distant metastasis. This raises the possibility that patients with invasive pituitary adenomas may have clinically silent metastasis, which would make pituitary carcinoma more common than generally is appreciated. Patients with invasive adenomas who show persistent hormone hypersecretion despite relatively radical neurosurgery may be candidates for further investigation of the presence of metastasis, or for treatment with systemic therapy.

In virtually all reported cases of pituitary carcinoma, the patients' presenting symptoms were related to the mass effect of the primary tumor. With the exception of patients with Cushing's disease, the course was one of progressively expanding intracranial mass, with average survival, after onset of symptoms, of approximately 4 years. In the presence of distant metastasis, surgical treatment has primarily been directed at decompression of neural structures adjacent to the primary tumor.

\section{Management}

Since local mass effects are the most common mode of presentation and reason for death in patients with pituitary carcinoma, surgery is often indicated to provide diagnosis and relief of symptoms. Modern cranial base techniques, which allow the best chance for maximum tumor debulking, may provide control of the local disease. The procedure may need to be supplemented with local radiotherapy. As is true in the present case and in 24 of the previously reported cases in the literature, prior radiotherapy had been given; this raises the issue of whether radiotherapy may have been an etiologic factor in the malignant transformation of these tumors. Nonetheless, radiation is effective therapy in the control of residual functioning and nonfunctioning tumors. ${ }^{17-19}$

The dopamine antagonist, bromocriptine, has been shown to be a effective treatment in most cases of nonmetastatic prolactinoma. ${ }^{20-24}$ However, of the eight reported cases of metastatic prolactinoma in the literature, six of the patients received bromocriptine treatment; three of these initially showed a good response and one showed continued remission. Seven of the eight patients eventually died of progressive disease. ${ }^{3}$ In view of the nature of the effect of bromocriptine, that is, the induction of cellular atrophy, rather than cell death, ${ }^{25}$ the persistence of tumor and even further growth are not surprising. Progressive malignant transformation in such a neoplasm might result in the loss of markers of differentiation, such as hormone receptors or biochemical mechanisms responsible for physiologic modulation, or the loss of response to cytostatic drugs. ${ }^{26}$ Such dedifferentiation may have occurred in our present case, as suggested by: (1) loss of immunoreactivity in a metastatic lesion compared with previous resection specimens, and (2) the ultrastructural features of this tumor displaying a poorly differentiated tumor whose cellular derivation could not readily be established. 
Bromocriptine may also be effective therapy in some patients with growth hormone-producing adenomas 27,28 and others secreting growth hormone and prolactin. ${ }^{23} \mathrm{~A}$ beneficial response to bromocriptine is reportedly more likely if an elevated serum prolactin is present, an elevation of plasma growth hormone occurs after thyroid releasing hormone (TRH) administration, plasma growth hormone falls $50 \%$ after a single test dose of bromocriptine, or an exaggerated prolactin response to TRH is recorded. ${ }^{27}$ In some cases, it appears that clinical improvement following bromocriptine does not always imply tumor shrinkage or improvement of biochemical parameters. ${ }^{29,30}$ Salti and Istifan ${ }^{31}$ reported on three women who showed clinical improvement and normalization of growth hormone with bromocriptine despite increasing sellar size. It remains difficult to drawn conclusions about the usefulness of bromocriptine in the treatment of these patients.

The presence of growth hormone receptors in acromegalic patients with pituitary adenomas has led to the use of several long-acting somatostatin analogues. These somatostatin analogues (SMS 201-995) have resulted in normal growth hormone and IGF-1/SMC levels after 6 months to 2 years in 50 to $75 \%$ of acromegalic patients, as well as a decrease in pituitary tumor size. ${ }^{32,33}$ This analogue may be effective in treatment of pituitary carcinoma with widespread metastasis.

The effect of hormonal treatment on nonsecreting tumors is far less satisfactory. Their effectiveness in the treatment of pituitary carcinoma remains questionable, especially since 14 of the patients reported in the literature died of progressive primary disease, and the majority of metastasis remained clinically silent. A reasonable management plan, in a patient with invasive pituitary adenomas, would be to exclude distant metastasis, perform radical local surgery wherever possible, with hormonal therapies or local radiotherapy, or both. Systemic metastasis would get direct attention either with surgery or radiotherapy if they became symptomatic or were in danger of becoming symptomatic, as might be the case in an unstable but asymptomatic spinal lesion.

\section{CONCLUSION}

The present case underscores the difficulty in distinguishing pituitary adenomas from carcinomas without the evidence of distant metastasis. Histologic overlap has been well known, and the observation of atypism and pleomorphism cannot be used for diagnosis or to predict behavior. Furthermore, even with the use of electron microscopy, immunohistochemical markers for pituitary hormones, and proliferation markers, no clear distinction can be made. Of interest in this case was the long duration between the initial presentation and the multiple recurrences. The patient also had multiple cranial base sur- the neoplasm malignant from the beginning? Did it dedifferentiate due to external factors such as radiation? Or are the metastases merely representations of embolization secondary to multiple surgical manipulations? These questions pose interesting problems for future investigation.

\section{REFERENCES}

1. Mountcastle RB, Roof BS, Mayfield RK, et al: Case report: Pituitary adenocarcinoma on acromegalic patient: Response to bromocriptine and pituitary testing: A review of the literature on 36 cases of pituitary carcinoma. Am J Med Sci 298:109-118, 1989

1a. Asai A, Matsutani M, Funada N, Takakura K: Malignant growth hormone-secreting pituitary adenoma with hematogenous dural metastasis: Case Report. Neurosurgery 22:1091-1094, 1988

lb. Cohen DL, Diengodh JV, Thomas DGT, Himsworth RL: An intracranial metastasis from a prolactin-secreting pituitary tumor. Clin Endocrinol 18:259-264, 1983

1c. Graff CJ, Blinerman EE, Terplan KL: Pituitary carcinoma in a child with distant metastasis. J Neurosurg 19:254-259, 1962

1d. Hashimoto $\mathrm{N}$, Harida $\mathrm{H}$, Nishi $\mathrm{S}$ : Intracranial and intraspinal dissemination from a growth hormone-secreting pituitary tumor. Case report. J. Neurosurg 64:140-144, 1986

le. Kuroki M, Tanaka R, Yokoyama M, Shimbo Y, Kiuta F: Subarachnoid dissemination of a pituitary adenoma. Surg Neurol 28:71-76, 1987

If. Nudleman KL, Choi B, Kussbe JA: Primary pituitary carcinoma: A clinical pathological study. Neurosurgery 16:90-95, 1985

1g. Plangger CA, Twerdy K, Grunert V, Weiser G: Subarachnoid metastases from a prolactinoma. Neurochirurgia 28:235-237, 1985

lh. Sang H, Johnson C: Metastatic prolactin-secreting pituitary adenoma. Hum Pathol 15:94-96, 1984

2. Nawata H, Higuchi K, Ikuyama S, Kato K, Ibayashi H, Mimura K, Sueshi $\mathrm{K}$, Zingami $\mathrm{H}$, Imura $\mathrm{H}$ : Corticotropin releasing hormones and adrenocorticotropin-producing pituitary carcinoma with metastasis to the liver and lung in a patient with Cushing disease. J Clin Endocrinol Metab 71:1068-1073, 1990

3. Popovic EA, Vattuone JR, Siu KH, Busmanis I, Pullar MJ, Dowling J: Malignant prolactinomas. Neurosurgery 29:127-130, 1991

4. Landolt AM: Ultrastructure of human sellar tumors. Acta Neurochir (Wien) 33 (Suppl 22):1-94, 1975

5. Kovacs K, Horvath E: Tumors of the pituitary gland. In Atlas of Tumor Pathology. Second Series Fascicle 21. Washington, DC: Armed Forces Institute of Pathology, 1986

6. Jefferson G: Extrasellar extensions in pituitary adenomas. Proc $\mathbf{R}$ Soc Med 33:433-458, 1940

7. Souther D, Saeger W: Invasiveness of pituitary adenomas. Pathol Res Pract 187:632-636, 1991

8. Madonick MJ, Rubinstein LJ, Dasio MR, Ribner H: Chromophobe adenoma of the pituitary gland with subarachnoid metastasis. Neurology 13:836-840, 1963

9. Ogilvy KM, Jakubowski J: Intracranial dissemination of pituitary adenomas. J Neurol Neurosurg Psychiatry 36:199-205, 1973

10. Nelson PB, Robinson AG, Martinez AJ: Metastatic tumor of the pituitary gland. Neurosurgery 21:941-944, 1987

11. Chiang MF, Brock M, Patt S: Pituitary metastasis. Neurochirurgia 33:127-131, 1990

12. Post KD, McCormick PC, Haze AP, Kandji AG: Metastatic carcinoma to the pituitary. Report of two cases. Surg Neurol 30 286-292, 1988

13. James RL, Jr., Arsenis G, Stoler M, Nelson C, Barn D: Hypophyseal metastatic renal cell carcinoma and pituitary adenoma. Case report and review of the literature. Am J Med 76:337-340, 1984

14. Tubiana M, Pejovic MH, Koscielny S: Growth rate, kinetics of tumor cell proliferation and long-term outcome in human breast cancer. Int J Cancer 44:17-22, 1989

15. Nishizaki T, Orita T, Furtani Y, et al: Flow cytometric DNA analysis and immunohistochemical measurement of $\mathrm{Ki}-67$ and BudR labeling indices in human brain tumors. J Neurosurg 70: 379-384, 1989 
16. Hsu DW, Hakim F, Biller BM, Monte S, Zervas NT, Klibanski A, Whyte T: Significance of proliferating cell antigen index in predicting pituitary carcinoma recurrence. J Neurosurg 78: 753-761, 1993

17. Ciric I, Mikhael M, Stafford T, Lawson L, Garces R: Transphenoidal microsurgery of pituitary macroadenomas with long term followup results. J Neurosurg 59:395-401, 1983

18. Flickinger JC, Nelson PB, Martinez AJ, Deutsch M, Taylor F: Radiotherapy of nonfunctional adenomas of the pituitary gland. Results with long term follow up. Cancer 63:2409-2414, 1989

19. Grigsby PW, Simpson JR, Fineberg B: Late regrowth of pituitary adenomas after irradiation and/or surgery. Cancer 63:13081312, 1989

20. Konopka P, Raymond JP, Merceron RE, Seneze J: Continuous administration of bromocriptine in the prevention of neurological complications in pregnant women with prolactinomas. Am J Obstet Gynectol 146:935-938, 1983

21. Lamberts SWJ: Antimitotic actions of dopaminergic drugs on human pituitary tumours. Neuroendocrinol Perspect 3:317343, 1984

22. Maurer RA: Dopaminergic inhibition of protein synthesis and prolactin mRNA accumulation in cultured pituitary cells. J Biol Chem 255:8092-8097, 1980

23. Weiss MH, Wycoff R, Yadley R, Gott P, Feldon S: Bromocryptine treatment of prolactin secreting tumors: Surgical implications. Neurosurgery 12:640-642, 1983

24. Mori H, Maeda T, Saitoh Y, Onishi T: Changes in prolactinomas and somatotropinomas in humans treated with bromocriptine. Pathol Res Pract 183:580-583, 1988

25. Tindall GT, Kovacs K, Horvath E, Thorner MO: Human prolactin- producing adenomas and bromocriptine: A histological immunocytochemical, ultrastructural, and morphometric study. J Clin Endocrinol Metab 55:1078-1083, 1982

26. Scheitauer BW, Randall RV, Laws ER, Kovacs KT, Horvath E, Whitaker MD: Prolactin cell carcinoma of the pituitary: Clinicopathologic, immunohistochemical, and ultrastructural study of a case with extracranial and intracranial metastasis. Cancer 55:598-604, 1985

27. Melmed S, Braunstein GD, Chang RJ, Beeker DP: Pituitary tumors secreting growth hormones and prolactin. Ann Intern Med 105 : 238-253, 1986

28. Wollesen F, Anderson T, Karle A: Size reduction of extracellular pituitary tumors during bromocriptine therapy. Quantitation of the effect on different types of tumors. Ann Int Med 96:281286, 1982

29. Besser GN, Wassjah, Thorner MO: Acromegaly results of longterm treatment with bromocriptine. Acta Endocrinol 88:187, 1978

30. Nillsson A: Experiences with bromocriptine treatment and acromegaly in preliminary report. Acta Endocrinol 88(Suppl):216215,1978

31. Salti IS, Istafan N: Bromocriptine fails to stop growth of iosinophilic adenomas and acromegaly. N Engl J Med 301:386, 1979

32. Barkan AL, Kelch RP, Hopwood NG, Beitins F: Treatment of acromegaly with long-acting somatostatin and analogue SMS 201-995. J Endocrinol Metab 66:16, 1988

33. Lamberts SWJ, Uitterlinden P, Del Pozo E et al.: SMS 201-995 induces a continuous decline in circulating growth hormone in somatostatin during therapy of acromegalic patients for over two years. J Clin Endocrinol Metab 65:703-710, 1987 